\title{
Label-Free Analysis and Sorting of Microalgae and Cyanobacteria in Microdroplets by Intrinsic Chlorophyll Fluorescence for the Identification of Fast Growing Strains
}

\author{
Roshni J. Best, ${ }^{\dagger, \dagger}$ Jan J. Lyczakowski, ${ }^{\dagger}$ Sara Abalde-Cela, ${ }^{\dagger}$ Ziyi Yu, ${ }^{\dagger}$ Chris Abell, ${ }^{*}{ }^{\dagger}$ and Alison G. Smith ${ }^{*}$, \\ ${ }^{\dagger}$ Department of Chemistry, University of Cambridge, Lensfield Road, Cambridge, CB2 1EW, U.K. \\ ${ }^{\ddagger}$ Department of Plant Sciences, University of Cambridge, Downing Street, Cambridge CB2 3EA, U.K.
}

Supporting Information

\begin{abstract}
Microalgae and cyanobacteria are promising organisms for sustainable biofuel production, but several challenges remain to make this economically viable, including identification of optimized strains with high biomass productivity. Here we report on a novel methodology for the labelfree screening and sorting of cyanobacteria and microalgae in a microdroplet platform. We show for the first time that chlorophyll fluorescence can be used to measure differences in biomass between

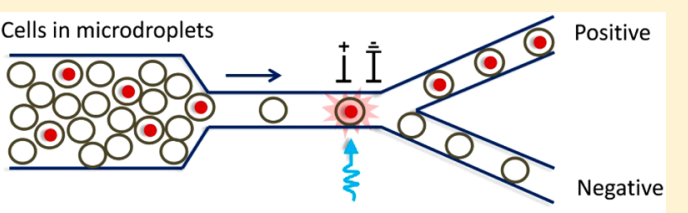

Microfluidic sorting based on chlorophyll fluorescence populations of picoliter microdroplets containing different species of cyanobacteria, Synechocystis PCC 6803 and Synechococcus PCC 7002, which exhibit different growth dynamics in bulk culture. The potential and robustness of this label-free screening approach is further demonstrated by the screening and sorting of cells of the green alga Chlamydomonas reinhardtii encapsulated in droplets.
\end{abstract}

$\mathrm{T}$ he emerging platform of microdroplets in microfluidics has been applied to study the growth and behavior of individual cells, including microalgae and cyanobacteria., These organisms can fix atmospheric carbon dioxide into biomass via oxygenic photosynthesis and have been proposed as suitable feedstocks for industrial purposes for a number of reasons. Cultivation of algae does not compete with agricultural crops for land. They require less water than land crops and could use waste $\mathrm{CO}_{2}$ from other industrial processes as a carbon source. ${ }^{3}$ Many microalgae and cyanobacteria have fast growth rates, and some have the potential to make significant levels of both high value and commodity products that can be sold commercially, ${ }^{4,5}$ e.g., the feasibility of producing biodiesel and bioethanol from algae is being investigated. ${ }^{6}$ However, many challenges remain to make these processes economically viable, and key areas for biological study include strain selection, optimization of growth conditions, and understanding of cellular metabolism. ${ }^{7}$ This requires a reliable system to screen and identify candidate microalgal species ${ }^{7}$ and strains that can subsequently be tested in large scale bioreactors. There is considerable effort worldwide to screen natural populations for species with suitable characteristics as well as programs to alter metabolism by genetic manipulation. ${ }^{8}$ Coupling these approaches to studying and screening microalgae at the singlecell level will increase throughput and enable hundreds or thousands of potential candidates to be screened, allowing identification of individual cells with appropriate biotechnological characteristics to be found from within a population.

Microfluidic devices have attracted significant attention over the past decade for their utility in chemical and biological analyses. ${ }^{9,10}$ For many cell studies, it can be advantageous to encapsulate cells within microdroplets in a microfluidics channel, and such systems are increasingly being used to study a wide variety of biological processes. ${ }^{11,12}$ Recent advances in microdroplet technology have employed fluorescence polarization, ${ }^{13,14}$ fluorescence-resonance-energy transfer (FRET), ${ }^{15,16}$ ultraviolet-visible spectroscopy (UV-vis), ${ }^{17}$ and electrochemical properties ${ }^{18-20}$ of individual cells for detection in high-throughput screening experiments. Effective detection within microfluidic environments is defined by a close interrelationship of factors such as detector sensitivity, response times, detection limits, and information content. ${ }^{21}$

Microdroplet sorting technology combines highly sensitive detection in microdroplets and high throughput manipulations to separate droplets of interest into a separate stream from the rest of the population. Methods have been established to allow sorting of droplets using laser-induced heating, ${ }^{22}$ "opticaltweezers", ${ }^{23}$ magnetic fields, ${ }^{24}$ acoustic waves, ${ }^{25}$ and valves. ${ }^{26,27}$ Droplets can also be passively sorted according to their size using channel geometry ${ }^{28}$ or by gravitational effects. ${ }^{29,30}$ Most microdroplet sorting is performed using an electric field that deflects droplets of interest via dielectrophoresis into a separate "positive" collection channel, while the rest of the droplet stream flows by default into a negative channel with lower hydrodynamic resistance. ${ }^{31}$ Laser-induced fluorescence (LIF) has provided by far the most interesting results in terms of sensitivity of detection and sorting ability in a microfluidic channel and microdroplets. In 2009, "fluorescence-activated droplet sorting" (FADS) was established by Baret et al. ${ }^{32}$ to

Received: June 19, 2016

Accepted: September 28, 2016

Published: September 28, 2016 
sort a mixed population of $E$. coli cells based on $\beta$-galactosidase enzymatic activity, which transformed a nonfluorescent $\beta$ galactoside substrate into fluorescein. Subsequently, the genotype and phenotype of encapsulated cells in droplets have been assessed by fluorescence in the whole droplet environment.

In contrast to the above, intrinsic chlorophyll fluorescence in photosynthetic cells is a label-free method to detect cells. It provides a direct indication of the photosynthetic activity of cells, since it is a reflection of whether there is efficient transfer of excitation energy through the photosynthetic apparatus. Enhanced chlorophyll fluorescence can be an indication of damage or cellular stress and has also been widely used to identify mutants with defects in one or more photosynthetic components. ${ }^{33}$ In fact, all chlorophyll-containing cells exhibit some level of fluorescence, and indeed this has been used as a measure of the number of cells present in a microdroplet. ${ }^{2}$ Herein, we extend this approach, and report on a microdroplet platform designed and developed to allow screening and sorting based on chlorophyll by monitoring natural autofluorescence in cells of two different cyanobacterial species and the green alga Chlamydomonas reinhardtii. The development and practical demonstration of this technique will allow for the label-free selection of fast growing strains.

\section{EXPERIMENTAL SECTION}

Device Fabrication. The microfluidic PDMS/glass devices were fabricated by conventional soft lithography methods. ${ }^{34}$ The design of the device was devised using Autocad 2007 (Autodesk), and a dark-field mask was printed (Microlitho). For the preparation of microfluidic devices with a channel depth of $25 \mu \mathrm{m}$, the procedure is as follows. SU-8 2025 photoresist (Micro-Chem) was spin-coated onto a silicon wafer (diameter, $76.2 \mathrm{~mm}$; Compart Technology Ltd.) at $500 \mathrm{rpm}$ for $5 \mathrm{~s}$ and $3000 \mathrm{rpm}$ for $40 \mathrm{~s}$. Thereafter the wafer went through a postapply baking process for $1 \mathrm{~min}$ at $65{ }^{\circ} \mathrm{C}$ and $2 \mathrm{~min}$ at 95 ${ }^{\circ} \mathrm{C}$. Subsequently, the spin-coated wafer was exposed to UV light through the mask on a mask aligner (MJB4, Suss Microtech) for $5 \mathrm{~s}$. After postbaking for $1 \mathrm{~min}$ at $65^{\circ} \mathrm{C}$ and 2 min at $95{ }^{\circ} \mathrm{C}$, the non cross-linked photopolymer was removed with propylene glycol monomethyl ether acetate (PMGEA) in order to develop the desired features on the master. Finally, the master was hard-baked for $1 \mathrm{~min}$ at $170{ }^{\circ} \mathrm{C}$. The steps for the fabrication of microfluidic devices with channel depth of $50 \mu \mathrm{m}$ are the same than described for $25 \mu \mathrm{m}$, with the following conditions: $500 \mathrm{rpm} 5 \mathrm{~s}$ and $1450 \mathrm{rpm} 40 \mathrm{~s}$ (spin-coating); 2 min at $65{ }^{\circ} \mathrm{C}$ and $6 \mathrm{~min}$ at $95{ }^{\circ} \mathrm{C}$ (postbaking); $7.5 \mathrm{~s}$ (UVexposure); $1 \mathrm{~min}$ at $65{ }^{\circ} \mathrm{C}$ and $3 \mathrm{~min}$ at $95{ }^{\circ} \mathrm{C}$ (postexposure baking). For the fabrication of the PDMS mold, a mixture of poly(dimethylsiloxane) (PDMS, Sylgard 184) and cross-linker (curing agent, Sylgard 184) (ratio 10:1, w/w) was poured over the silicon master and degassed for $30 \mathrm{~min}$ before curing it overnight at $75{ }^{\circ} \mathrm{C}$. The cured device was cut and peeled from the master, and holes for tubing were made with a biopsy punch (i.d. $=0.38 \mathrm{~mm}$ ). After treatment with oxygen plasma for $6 \mathrm{~s}$ at oxygen pressure $1 \mathrm{bar}$, the device was bound to a glass slide in order to seal the microfluidic system. The device was baked at $90{ }^{\circ} \mathrm{C}$ for $1 \mathrm{~h}$ to make the sealing permanent. Finally, the microfluidic channels were treated with Aquapel (Pittsburgh, PA), a commercially available fluorosilane, followed by flushing of the channels with fluorous oil and nitrogen for drying.
The fabrication of the sorting devices required inserting electrodes into extra channels in the PDMS mold. This involved filling such channels with $51 \mathrm{In} / 32.5 \mathrm{Bi} / 16.5 \mathrm{Sn}$ lowtemperature solder (Indium Corporation) by melting a small piece of the conductive material inside the channels while keeping the device at $140{ }^{\circ} \mathrm{C}$ on a heating plate. ${ }^{32,35}$ For electrical connections, short pieces of electrical wire connected to the solder electrodes were used. The dimensions and design of the devices used in this work are specified in each of the corresponding sections for encapsulation, detection, and sorting for the different species.

Cell Culture Conditions. C. reinhardtii were cultured in TAP (Tris-Acetate-Phosphate) medium $^{36}$ using the trace elements as described by Kropat et al. ${ }^{37}$ Microalgae were routinely cultured in $100 \mathrm{~mL}$ conical flasks at room temperature under continuous illumination of $80 \mu \mathrm{mol}$ of photons $\mathrm{m}^{-2} \mathrm{~s}^{-1}$ and shaken at $150 \mathrm{rpm}$.

Cyanobacteria Synechocystis PCC 6803 and Synechococcus PCC 7002 were cultured in $25 \mathrm{~mL}$ of sterile BG11 medium supplemented with $0.1 \mathrm{M} \mathrm{NaHCO}_{3}$ to enhance cyanobacterial growth. ${ }^{38}$ As Synechococcus PCC 7002 is auxotrophic for cobalamin, the vitamin was supplemented in the medium to a concentration of $500 \mathrm{pM}$. Disposable sterile flasks (ThermoScientific), allowing for air exchange, were used for cyanobacterial growth. Cultures were incubated at $30{ }^{\circ} \mathrm{C}, 120$ rpm with constant $80 \mu \mathrm{mol}$ of photons $\mathrm{m}^{-2} \mathrm{~s}^{-1}$ illumination.

To prepare cells for encapsulation into microdroplets, a $1 \mathrm{~mL}$ sample of midexponential phase cells was taken, centrifuged for $3 \mathrm{~min}$ at $1300 \mathrm{rpm}$ using an Eppendorf MiniSpin centrifuge, resuspended in fresh growth media and diluted to the desired cell concentration for encapsulation into droplets (details given in Cell encapsulation section). Microalgae concentrations were determined by direct counting using a bright-line hemocytometer (Sigma-Aldrich) and bacterial culture growth and optical density was measured at $730 \mathrm{~nm}$ using a spectrophotometer (WPA Biochrome).

Cell Encapsulation in Droplets. A suspension of $\sim 5 \times$ $10^{6} \mathrm{C}$. reinhardtii cells $\mathrm{mL}^{-1}$ in TAP growth media was injected at $600 \mu \mathrm{L} \mathrm{h}^{-1}$ as the aqueous phase into a flow-focusing device of dimensions $40 \mu \mathrm{m} \times 25 \mu \mathrm{m}$ (width $\times$ depth). The oil phase (HFE-7500 fluorinated oil containing 3\% PicoSurf 1 surfactant, Sphere Fluidics) was injected at $1400 \mu \mathrm{L} \mathrm{h}^{-1}$. Monodisperse 40 $\mu \mathrm{m}$ diameter (34 pL volume) droplets were generated.

Flow-focusing geometry devices of dimensions $30 \mu \mathrm{m} \times 50$ $\mu \mathrm{m}$ (width $\times$ depth) were used to encapsulate Synechocystis PCC 6803 and Synechococcus PCC 7002 into aqueous microdroplets. A suspension of the cyanobacterial culture of OD730 $=0.03\left(1.8 \times 10^{6}\right.$ cell $\left.\mathrm{mL}^{-1}\right)$ was used as an aqueous phase. FC-40 oil (3M) containing $2.5 \%$ of a fluorosurfactant (PicoSurf2, Sphere Fluidics) was used as a dispersed phase. Liquids were pumped into a flow-focusing device via $1 \mathrm{~mm}$ polyethylene tubing (Smiths). Oil and cyanobacterial cultures were delivered with a syringe and the infusion process was carried out by automated pressure pumps (Harvard Apparatus) at $2000 \mu \mathrm{L} \mathrm{h}^{-1}$ and $200 \mu \mathrm{L} \mathrm{h}^{-1}$, respectively. Droplet size (50 $\mu \mathrm{m}$ diameter) and quality was monitored during the manufacturing process using an inverted microscope (Olympus IX71) equipped with a fast camera (Phantom Miro-4, Vision Research).

Optical Setup for Detecting Chlorophyll Fluorescence. To detect fluorescence in the microdroplet sorting setup, fixed wavelength lasers are used for excitation, and the ones available (Cobolt) had fixed wavelengths of $488 \mathrm{~nm}$ of 20 

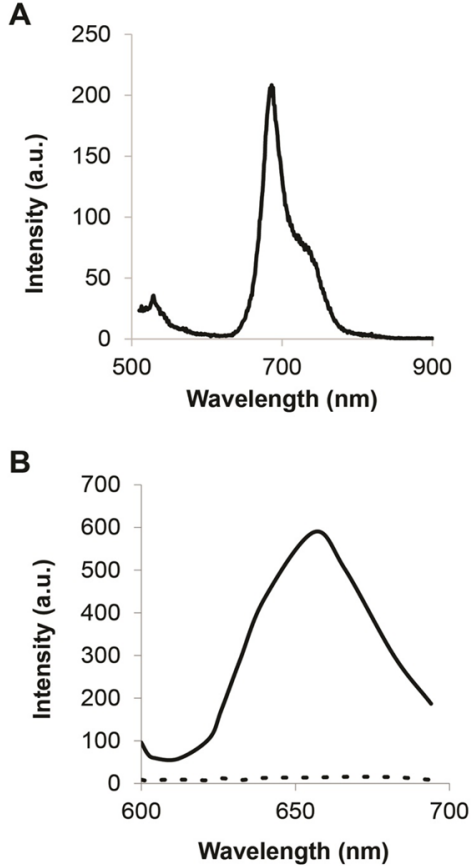

C

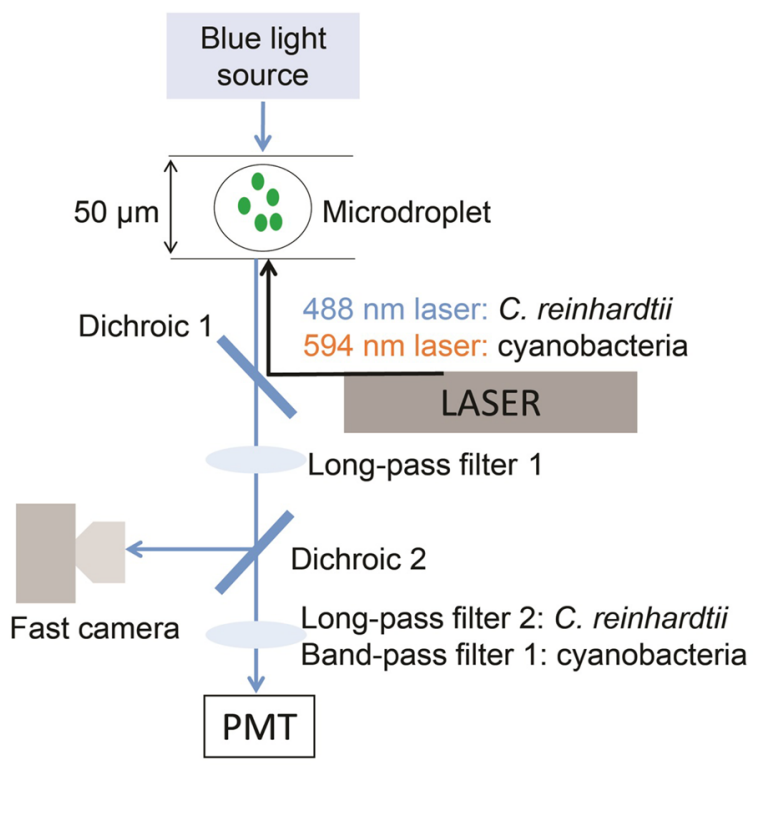

Figure 1. (A) Fluorescence emission profiles after excitation with $488 \mathrm{~nm}$ laser source for C. reinhardtii; (B) fluorescence emission profiles for cyanobacteria after excitation with $594 \mathrm{~nm}$ (continuous line) and $488 \mathrm{~nm}$ (discontinuous line) laser source; (C) fluorescence screening and sorting setup for C. reinhardtii and cyanobacteria Synechocystis PCC 6803 and Synechococcus PCC 7002. Long-pass filter $1=360 \mathrm{~nm}$; dichroic $1=488 \mathrm{~nm}$ (for C. reinhardtii) and $594 \mathrm{~nm}$ (for cyanobacteria); dichroic $2=633 \mathrm{~nm}$; long-pass filter $2=635 \mathrm{~nm}$; band-pass filter $1=629 \pm 30 \mathrm{~nm}$.

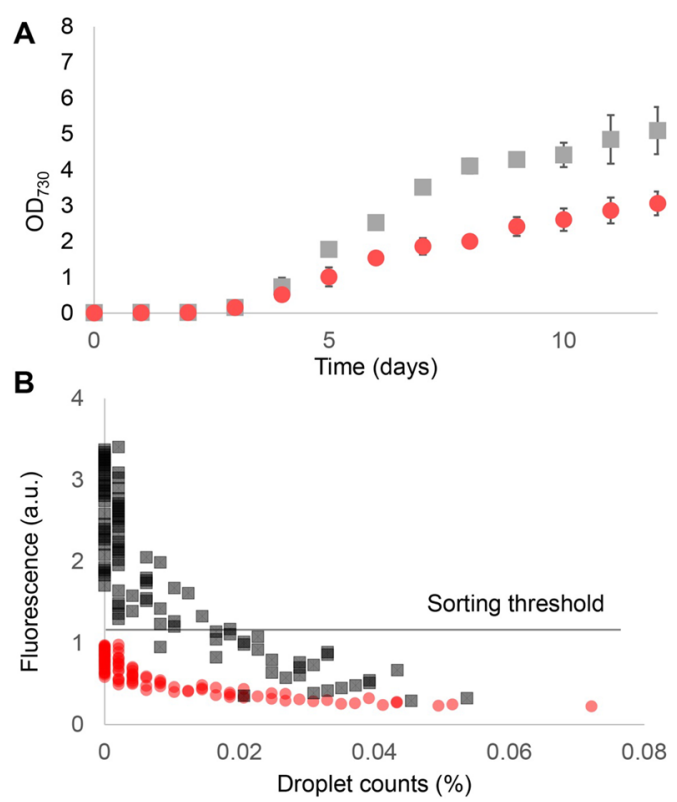

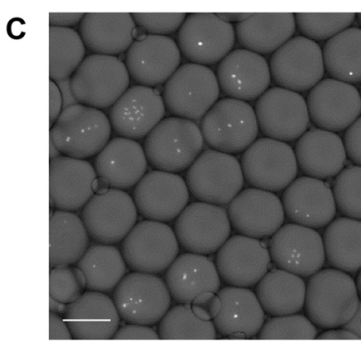

4 days incubation PCC6803

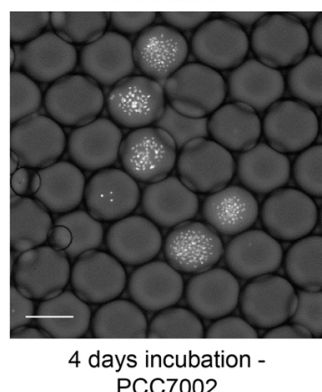

Figure 2. (A) OD730 for Synechocystis PCC 6803 (red circles) and Synechococcus PCC 7002 (gray squares) bulk cultures measured over 3 weeks; (B) plot of the fluorescence intensity per droplet (500 droplets data) for the two different species (6803, red circles; 7002, gray squares). The line indicates the sorting threshold and how droplets with higher biomass can be sorted; (C) fluorescence images showing cyanobacteria intrinsic chlorophyll fluorescence after 4 days. Scale bar $=50 \mu \mathrm{m}$.

$\mathrm{mW}$ and $594 \mathrm{~nm}$ of $50 \mathrm{~mW}$. To establish which would be appropriate for the different species, chlorophyll fluorescence spectra were measured in a fluorimeter (Agilent Technologies) with excitation at these wavelengths. For C. reinhardtii, the 488 $\mathrm{nm}$ laser resulted in efficient excitation (Figure 1A), but for both the cyanobacterial species this did not result in any fluorescence. Instead, $594 \mathrm{~nm}$ excitation was effective (Figure 1B) and so was used for Synechocystis PCC 6803 and
Synechococcus PCC 7002. The fluorescence spectra of both PCC 6803 and PCC 7002, obtained using this excitation frequency, were essentially the same intensity (Supporting Information Figure S1), indicating that the excitation method will generate no bias toward one of the species analyzed.

Fluorescence detection for sorting of microdroplets was carried out by a multipart optical instrument. Figure 1C details the optical components used. For the in-flow detection and 

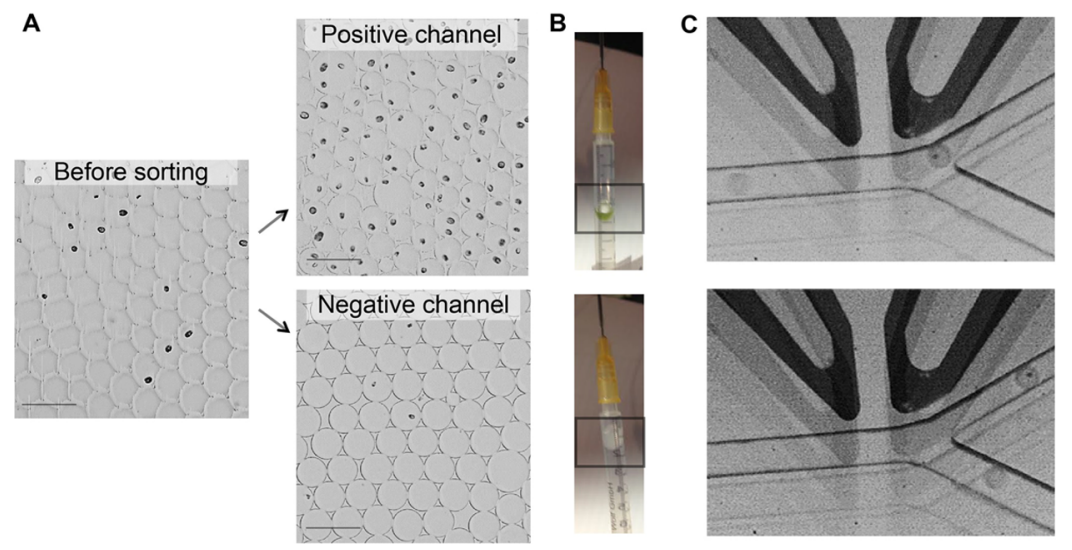

Figure 3. Sorting C. reinhardtii cell-containing droplets from empty droplets. (A) Images taken of droplets before and after sorting. All droplets collected in the "positive" channel contain cells whereas only $3 \%$ of negative droplets are occupied, false negatives. Scale bar $=100 \mu \mathrm{m}$. Inset: A layer of green cell-containing droplets is collected from the "positive" channel in a syringe (top), whereas droplets collected from the "negative" channel are colorless (bottom). (C) Images recorded during sorting show that cell-containing droplets are deflected into the "positive" channel (top panel), whereas empty droplets flow into the "negative" channel (bottom panel).

sorting, droplets were reinjected into a sorting device with channel dimensions of $60 \mu \mathrm{m} \times 50 \mu \mathrm{m}(\mathrm{w} \times \mathrm{h})$, with a rate of $50 \mu \mathrm{L} \mathrm{h}^{-1}$ and spaced with HFE-7500 (3M) oil flow at $500 \mu \mathrm{L}$ $\mathrm{h}^{-1}$. Droplet frequency was monitored using a fast camera (Phantom Miro-4, Vision Research). The obtained fluorescence signal was saved onto a data acquisition card (National Instruments). Chlorophyll-associated fluorescence peaks were identified and analyzed using a customized script in LabView 8.2 software (National Instruments). In parallel, the fluorescence from each of the droplets passing through the laser path signal was detected by a photomultiplier (PMT). After a fluorescence read-out over a specific threshold defined by the transistor-transistor logic gate gate (TTL), the pulse generator sent a signal to the high voltage amplifier (HVA), which triggered the electrodes in the chip, deflecting the droplet to the "positive" channel. Cells in droplets were sorted at $300 \mathrm{~Hz}$. Droplets from the "positive" and "negative" channels were collected in $1 \mathrm{~mL}$ syringes for further analysis.

\section{RESULTS AND DISCUSSION}

Determining Biomass within Synechocystis PCC 6803 and Synechococcus PCC 7002 Containing Droplets. Chlorophyll fluorescence has been used to determine the number of algal cells in droplets to follow the growth kinetics; ${ }^{2}$ however, this was a static system. As proof of concept for the use of chlorophyll fluorescence to monitor an increase in cell numbers in droplets, we used populations of similar cells, but which exhibit different characteristics. Cyanobacteria Synechocystis PCC 6803 and Synechococcus PCC 7002 were selected due to their different growth rates and final cell densities observed in bulk culture experiments (Figure 2A). Synechococcus PCC 7002 cells are rod-shaped and approximately $1 \mu \mathrm{m} \times 2 \mu \mathrm{m}$. This species was chosen as a model for a fast growing species with a doubling time in bulk cultures $(\mu)$ of $17 \mathrm{~h}$ and a final carrying capacity $(\mathrm{K})$ of 4.4 as determined by adjusting the data to a logistic function (MatLab). In contrast, Synechocystis PCC 6803 (spherical, average diameter $1 \mu \mathrm{m}$ ) is slower growing, with a determined $\mu=21 \mathrm{~h}$ and $\mathrm{K}=2.5$. Populations of each species were separately encapsulated in droplets of $50 \mu \mathrm{m}$ and held in a syringe for 4 days at $30{ }^{\circ} \mathrm{C}, 80 \mu \mathrm{mol}$ photons $\mathrm{m}^{-2} \mathrm{~s}^{-1}$ illumination. The average number of cells per droplet (cdp) was determined by counting the number of cells in each droplet using chlorophyll fluorescence images taken in a static microscope (Figure S2 and Table S1, Supporting Information). The cells were viable and divided readily. The results confirmed that Synechococcus PCC 7002 grew faster, generating significantly higher biomass in an average droplet than Synechocystis PCC 6803 after 4 days (Figure $2 \mathrm{C}$ ).

At 4 days, droplets were then injected into a detection device at $50 \mu \mathrm{L} \mathrm{h}^{-1}$ and spaced with oil at $500 \mu \mathrm{L} \mathrm{h}^{-1}$. The droplets were flowed past a $594 \mathrm{~nm}$ laser, followed by detection of the fluorescent signal recorder by a PMT for $30 \mathrm{~s}$. Figure 2B shows the frequency of droplet counts versus the level of fluorescence intensity. Clearly, the Synechocystis PCC 6803 cells on average have a much lower fluorescence than the Synechococcus PCC 7002 cells. The signal gain value for the lower occupancy Synechocystis PCC 6803 droplets was adjusted so that none of the fluorescence peaks exceeded the detection threshold. When the same setting was used for the analysis of Synechococcus PCC 7002-containing droplets 175 out of 1950 droplets passing the laser for $30 \mathrm{~s}$ had chlorophyll fluorescence that exceeded the set detection threshold. When the droplet scan rate (65 droplets/ second) and percentage of cell containing droplets (15\%) are considered, this result indicates that approximately $60 \%$ of Synechococcus PCC 7002 droplets scanned had a detectable amount of biomass for the preset PMT gain. By analyzing the droplet growth data for the species (used to obtain average values in Table S1 and the histograms in Figure S2, Supporting Information), it was concluded that in this experimental setup all droplets containing more than 40 cyanobacterial cells gave a "positive" signal.

The results demonstrate that using in-flow laser-induced detection of chlorophyll fluorescence, it is possible to discriminate low cyano-bacterial cell occupancy droplets $(<40$ cells) from high occupancy ones ( $>40$ cells). This value could be adjusted by altering the signal gain value. Thus, the system is a versatile tool capable of discriminating the cyanobacterial biomass content in droplets. In principle it could be used for inflow discrimination of biomass. This can be achieved because fluorescence readings can be correlated with droplet growth. Synechococcus PCC 7002 containing droplets have higher cell counts than Synechocystis PCC 6803 ones after 4 days of in droplet growth (Figure S2, Supporting Information). Thus, enhanced fluorescence from PCC 7002 containing droplets was 

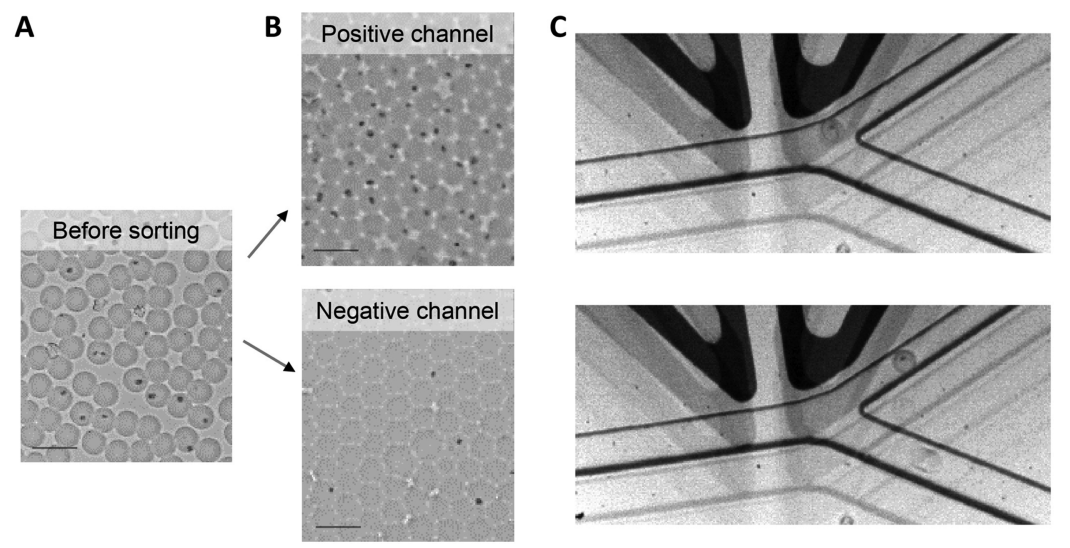

Figure 4. Sorting C. reinhardtii low-chlorophyll cell-containing droplets from empty droplets. (A) Images taken of droplets before sorting; (B) 91\% droplets collected in the "positive" channel contain cells whereas only $6 \%$ of negative droplets are occupied, false "negatives". Scale bar $=100 \mu \mathrm{m}$. (C) Images recorded during sorting show that cell-containing droplets are deflected into the "positive" channel (top panel), whereas empty droplets flow into the "negative" channel (bottom panel).

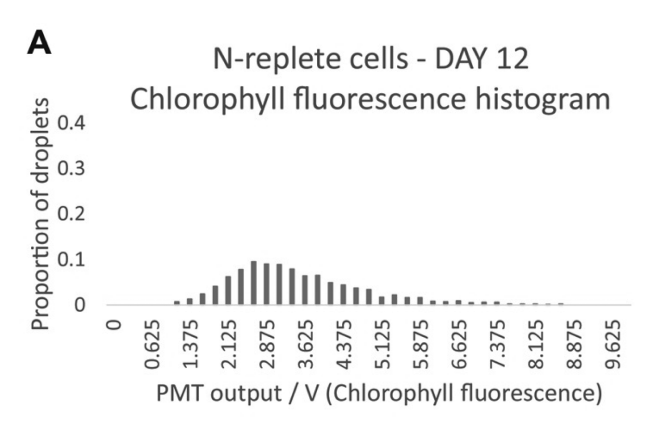

B

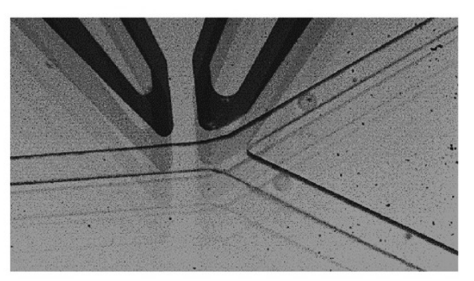

$\mathrm{N}$-limited cells - DAY 12

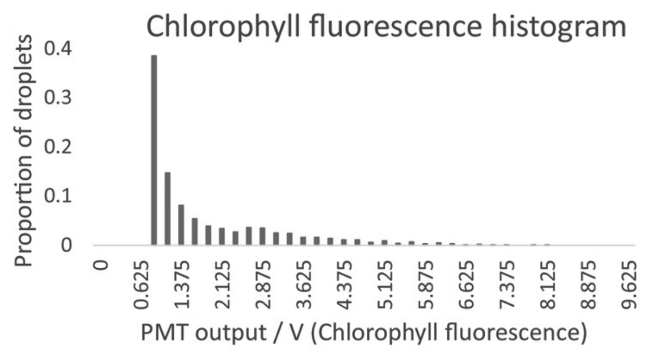

C

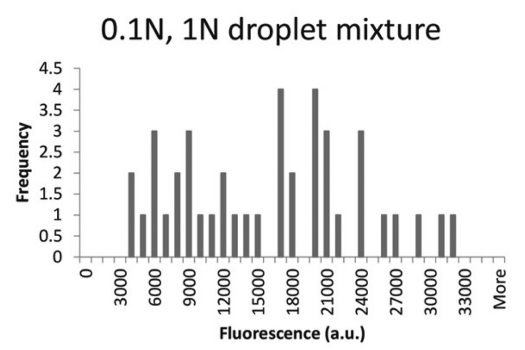

Negative channel, post sorting

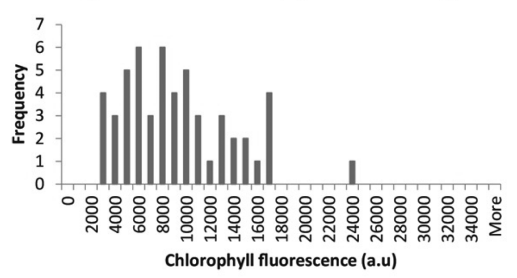

Figure 5. (A) Chlorophyll fluorescence profiles for cells grown in normal growth medium (1 N TAP, top panel) and nitrogen limited medium (0.1 $\mathrm{N}$ TAP, bottom panel). Droplets derived from C. reinhardti culture grown in normal medium have higher chlorophyll fluorescence. (B) Images collected during sorting: a droplet containing a high-chlorophyll cell is sorted into the "positive" channel (top panel) and an empty droplet and a droplet containing a low-chlorophyll cell are not sorted and flow into the "negative" channel (bottom panel). (C) The fluorescence of each cell was quantified from the collected images using ImageJ software. The fluorescence of cells from the negative channel matches the "low-fluorescence" cells from the droplet mixture.

indeed a measure of higher biomass content. As fluorescence is a reflection of physiological state and species identity, the instrument will need to be calibrated on a case by case basis to achieve good correlation between the fluorescence read-out and droplet biomass content.

Label-Free Sorting Based on Chlorophyll Fluorescence. We then extended the methodology to use chlorophyll fluorescence as a proxy for the presence of algal cells in droplets for high-throughput sorting using the green alga C. reinhardtii (average diameter $10 \mu \mathrm{m}$ ). Figure S3A, Supporting Information, shows the design of an experiment based on chlorophyll fluorescence to sort a mixture of empty and cell-containing droplets, to collect the cell-containing droplets in the "positive" sorting channel. We applied this method to C. reinhardtii cells from a culture grown in bulk in TAP media for 12 days. Cells were encapsulated in microdroplets of $40 \mu \mathrm{m}$ diameter, at a dilution designed to ensure one or no cells per droplet. After the encapsulation process approximately $14 \%$ of droplets contained one encapsulated cell and $1 \%$ of droplets contained more than one cell (Figure 3A). These frequencies match expected Poisson statistics. ${ }^{39,40}$ The droplets were loaded onto the sorting device at $30 \mu \mathrm{L} \mathrm{h}^{-1}$ and fluorinated carrier oil was injected at $300 \mu \mathrm{L} \mathrm{h}^{-1}$ to space the droplets out. As the droplets flowed past the laser spot, their intrinsic chlorophyll fluorescence was detected by the PMT and recorded on LabVIEW software. Each peak detected above the preset threshold triggered a "sorting event", where a voltage pulse (of $700 \mathrm{~V}$ ) was applied deflecting the droplet into the "positive" channel (Figure 3B); all other droplets passed into the "negative" channel. The number of expected peaks was 
calculated from the droplet encapsulation frequencies. Of these predicted peaks, $90 \%$ of them were detected, with $60 \%$ of the detected peaks above the sorting threshold. The pool of droplets was sorted at $300 \mathrm{~Hz}$ for $6 \mathrm{~h}$. Figure 3B shows that after sorting, all droplets from the positive collection syringe contained cells, no false positives were seen, and the pooled droplets appeared as a thin green layer in the collection syringe (Figure 3B, inset). In contrast, droplets collected in the negative collection syringe were colorless, although a small number $(\sim 3 \%)$ of false negatives were observed, that is, droplets containing cells. Figure 3C (top panel) shows a still image recorded during sorting in which the spherical $C$. reinhardtii cell can be seen in a droplet. This is deflected into the "positive" channel, whereas an empty droplet continues into the "negative" channel for a separate collection (Figure 3C, bottom panel). The full sorting video (Movie A) is available as Supporting Information.

Of particular note is that cells from both the "positive" and "negative" collection channels were motile in droplets after sorting, as the encapsulated cells can be seen swimming around (Figure S4, Supporting Information). This demonstrates that droplet manipulations during sorting, including the application of a voltage pulse to the droplets to deflect them into the sorting channel, did not appear to affect cell viability or motility. The sorting process was not destructive, and live cells were recovered for further analyses.

Sorting of High and Low Chl-Containing Cells. The ultimate aim of our microdroplet screening and sorting experiments was to be able to distinguish between droplets that exhibit different levels of fluorescence, to identify cells with desired fluorescence properties from within a population of cells, and sort them for further study. The experiment above used cells grown in media with sufficient nitrogen for normal growth (1 N TAP), and these had relatively high levels of chlorophyll. We prepared a culture of $C$. reinhardtii cells grown in nitrogen-limited medium (0.1 N TAP) and encapsulated them in droplets, aiming for no more than one cell per droplet. In this experiment, this resulted in $18 \%$ of droplets being occupied (Figure 4A). Encapsulated C. reinhardtii grown in nitrogen limited medium has much lower chlorophyll fluorescence levels than those grown in $1 \mathrm{~N}$ TAP (Figure $5 \mathrm{~A})$.This feature is a characteristic of microalgal cells subjected to nitrogen stress concomitant with an induction in neutral lipid (TAG) production. ${ }^{41}$ Droplets with low chlorophyll cells were reinjected into a microdroplet sorting chip as described in the previous section. As the droplets flowed past the laser, fluorescence above a threshold triggered a voltage pulse, which deflected the droplet into the positive channel. Droplets containing low-chlorophyll cells were collected in the positive channel. In this case, $91 \%$ of droplets deflected into the positive sorting channel contained one cell, and $9 \%$ of droplets were empty, i.e., false positives (Figure 4B). In the negative channel $6 \%$ of droplets were false negatives since they contained a cell (Figure 4B). Having shown the above, a mixture of droplets containing cells with high-chlorophyll (grown in $1 \mathrm{~N}$ TAP media) and those with low chlorophyll (grown in $0.1 \mathrm{~N}$ TAP media) were prepared in a 1:1 ratio (Figure S3B, Supporting Information). The mixed population of droplets was screened after reinjection into the sorting chip at the same flow rates used for the individual droplet population analysis. The gain on the LabView software was adjusted so that approximately half of the peaks corresponding to high-chlorophyll cells were above the sorting threshold, while peaks for low-chlorophyll cells were below this threshold. Droplets were sorted at $160 \mathrm{~Hz}$ for $6 \mathrm{~h}$ during which time 3.46 million droplets were sorted. Droplets containing high-chlorophyll cells triggered sorting and were deflected into the positive collection channel (Figure 5B top panel), and a total of 259000 droplets $(7.5 \%)$ were deflected into the positive channel. Empty droplets and droplets containing low-chlorophyll cells did not trigger sorting (Figure 5B bottom panel), so these were collected together in the negative sorting channel. In the starting mixed population, $15 \%$ of droplets contained cells, and 58\% of those triggered sorting, in other words were above the threshold. Assuming that all cells were detected, approximately half of the high chlorophyll cells were collected in the positive channel, The full sorting video (Movie B) is available in the Supporting Information.

After sorting, the droplets from the negative channel were reinjected and reimaged for chlorophyll fluorescence, followed by and quantification with ImageJ (Figure 5C, bottom panel). Clearly droplets in the negative channel have much lower fluorescence after sorting than those of the mixed population (Figure 5C, upper panel). Insufficient droplets were obtained in the positive channel to allow for reinjection and reimaging.

\section{CONCLUSIONS}

Herein, we present a novel method for the screening and sorting of cyanobacteria and microalgae in microdroplets. The endogenous chlorophyll fluorescence in photosynthetic organisms is directly related to physiological properties within the cell. This direct and label-free detection method does not require cell staining or fluorescence labeling of cells, avoiding tedious, time-consuming and expensive sample preparation steps, and ensures that the cells are as close as possible to their native physiological state.

The detection of chlorophyll fluorescence in cells encapsulated in droplets is shown to be possible and quantitative. Using two cyanobacterial species, Synechocystis PCC 6803 and Synechococcus PCC 7002, whose growth rates differ in bulk cultures, the system could discriminate between the cells of the two species after 4 days of growth in the microdroplets. With this as a basis, the next level of sophistication of the system was tested, C. reinhardtii was chosen because its size $(10 \mu \mathrm{m}$ diameter) allowed for an easier visualization when encapsulated in microdroplets. It was possible to demonstrate the same highthroughput screening based on chlorophyll fluorescence and to couple it to sorting in the device, enabling a mixed population of cells with different levels of chlorophyll to be separated. Significantly, the cells remained viable after the process, as demonstrated by their motility.

The results presented here represent a significant advance over previous studies, since the detection method is directly related to the physiological properties of each single-cell. Moreover, as chlorophyll levels tend to decrease under conditions of nitrogen depletion, ${ }^{41}$ which induces lipid biosynthesis in microalgae, so strains showing a rapid decline in chlorophyll fluorescence would indicate high lipid productivity. This technique is therefore suitable to be applied as a screening tool for microalgal libraries to select strains that would be suitable for production of these molecules. A second application comes from the fact that the method allows not only measurement of intrinsic chlorophyll per cell but also the total chlorophyll per droplet. The latter is a proxy for the amount of biomass per unit volume, thus making this technique a means to determine cell number and biomass evolution over 
time, both factors important to be optimized for industrial strains.

\section{ASSOCIATED CONTENT}

\section{S Supporting Information}

The Supporting Information is available free of charge on the ACS Publications website at DOI: 10.1021/acs.analchem.6b02364.

Fluorescence emission spectra for PCC 6803 and PCC 7002 (Figure S1), histograms visualizing droplet growth of Synechocystis PCC 6803 and Synechococcus PCC 7002 (Figure S2), diagrams for the experimental design of sections (Figure S3), images taken of droplets recovered from the positive and negative collection channels after sorting (Figure S4), number of cells per droplets for each species at day 4 after single-cell encapsulation (Table S1), and OD730 values of cultures used for encapsulation experiments (Table S2) (PDF)

Movie of sorting high $\mathrm{N}$ C. reinhardtii cells from empty droplets (AVI)

Movie of sorting low $\mathrm{N}$ C. reinhardtii cells from empty droplets (AVI)

\section{AUTHOR INFORMATION}

\section{Corresponding Authors}

*E-mail: ca26@cam.ac.uk.

*E-mail: as25@cam.ac.uk.

\section{Notes}

The authors declare no competing financial interest.

Additional data related to this publication is available at the University of Cambridge data repository (http://dx.doi.org/10. 17863/CAM.4309).

\section{ACKNOWLEDGMENTS}

Author R.J.B. was supported by a Doctoral Training Grant from the Engineering and Physical Sciences Research Council (EPSRC) of the UK (Reference EP/P505445/1), and author J.J.L. was in receipt of a studentship from the Biotechnology and Biological Sciences Research Council (BBSRC) of the U.K. as part of the Cambridge BBSRC-DTP Programme (Reference BB/J014540/1). Authors S.A.-C., C.A., and A.G.S. acknowledge funding from the EC within the FP7 DEMA project, Grant Agreement No. 309086, and authors Z.Y., C.A., and A.G.S. were in receipt of funding from BBSRC sLoLa Award Reference BB/L002957/1.

\section{REFERENCES}

(1) Abalde-Cela, S.; Gould, A.; Liu, X.; Kazamia, E.; Smith, A. G.; Abell, C. J. R. Soc., Interface 2015, 12 (106), 20150216.

(2) Pan, J.; Stephenson, A. L.; Kazamia, E.; Huck, W. T. S.; Dennis, J. S.; Smith, A. G.; Abell, C. Integr. Biol. 2011, 3, 1043-1051.

(3) Jones, C. S.; Mayfield, S. P. Curr. Opin. Biotechnol. 2012, 23, 346351.

(4) Cardozo, K. H. M.; Guaratini, T.; Barros, M. P.; Falcão, V. R.; Tonon, A. P.; Lopes, N. P.; Campos, S.; Torres, M. A.; Souza, A. O.; Colepicolo, P.; Pinto, E. Comp. Biochem. Physiol., Part C: Toxicol. Pharmacol. 2007, 146, 60-78.

(5) Pulz, O.; Gross, W. Appl. Microbiol. Biotechnol. 2004, 65, 635648.

(6) Scott, S. A.; Davey, M. P.; Dennis, J. S.; Horst, I.; Howe, C. J.; Lea-Smith, D. J.; Smith, A. G. Curr. Opin. Biotechnol. 2010, 21, 277286.
(7) Beer, L. L.; Boyd, E. S.; Peters, J. W.; Posewitz, M. C. Curr. Opin. Biotechnol. 2009, 20, 264-271.

(8) Georgianna, D. R.; Mayfield, S. P. Nature 2012, 488, 329-335.

(9) deMello, A. J. Nature 2006, 442, 394-402.

(10) Yin, H.; Marshall, D. Curr. Opin. Biotechnol. 2012, 23, 110-119.

(11) Joensson, H. N.; Andersson Svahn, H. Angew. Chem., Int. Ed. 2012, 51, 12176-12192.

(12) Rakszewska, A.; Tel, J.; Chokkalingam, V.; Huck, W. T. S. NPG Asia Mater. 2014, 6, e133.

(13) Choi, J.-W.; Kang, D.-K.; Park, H.; deMello, A. J.; Chang, S.-I. Anal. Chem. 2012, 84, 3849-3854.

(14) Joensson, H. N.; Zhang, C.; Uhlén, M.; Andersson-Svahn, H. Electrophoresis 2012, 33, 436-439.

(15) Rane, T. D.; Zec, H. C.; Puleo, C.; Lee, A. P.; Wang, T.-H. Lab Chip 2012, 12, 3341-3347.

(16) Srisa-Art, M.; Dyson, E. C.; deMello, A. J.; Edel, J. B. Anal. Chem. 2008, 80, 7063-7067.

(17) Dolega, M. E.; Jakiela, S.; Razew, M.; Rakszewska, A.; Cybulski, O.; Garstecki, P. Lab Chip 2012, 12, 4022-4025.

(18) Han, Z.; Li, W.; Huang, Y.; Zheng, B. Anal. Chem. 2009, 81, $5840-5845$

(19) Kemna, E. W. M.; Segerink, L. I.; Wolbers, F.; Vermes, I.; van den Berg, A. Analyst 2013, 138, 4585-4592.

(20) Niu, X.; Zhang, M.; Peng, S.; Wen, W.; Sheng, P. Biomicrofluidics 2007, 1, 044101

(21) Whitesides, G. M. Nature 2006, 442, 368-373.

(22) Baroud, C. N.; Delville, J.-P.; Gallaire, F.; Wunenburger, R. Phys. Rev. E 2007, 75, 046302

(23) Flynn, R. A.; Birkbeck, A. L.; Gross, M.; Ozkan, M.; Shao, B.; Wang, M. M.; Esener, S. C. Sens. Actuators, B 2002, 87, 239-243.

(24) Joung, J.; Shen, J.; Grodzinski, P. IEEE Trans. Magn. 2000, 36, 2012-2014.

(25) Schmid, L.; Weitz, D. A.; Franke, T. Lab Chip 2014, 14, 37103718.

(26) Abate, A. R.; Agresti, J. J.; Weitz, D. A. Appl. Phys. Lett. 2010, 96, 203509.

(27) Cao, Z.; Chen, F.; Bao, N.; He, H.; Xu, P.; Jana, S.; Jung, S.; Lian, H.; Lu, C. Lab Chip 2013, 13, 171-178.

(28) Tan, Y.-C.; Fisher, J. S.; Lee, A. I.; Cristini, V.; Lee, A. P. Lab Chip 2004, 4, 292-298.

(29) Maenaka, H.; Yamada, M.; Yasuda, M.; Seki, M. Langmuir 2008, $24,4405-4410$

(30) Yamada, M.; Nakashima, M.; Seki, M. Anal. Chem. 2004, 76, 5465-5471.

(31) Ahn, K.; Kerbage, C.; Hunt, T. P.; Westervelt, R. M.; Link, D. R; Weitz, D. A. Appl. Phys. Lett. 2006, 88, 024104.

(32) Baret, J.-C.; Miller, O. J.; Taly, V.; Ryckelynck, M.; El-Harrak, A.; Frenz, L.; Rick, C.; Samuels, M. L.; Hutchison, J. B.; Agresti, J. J.; Link, D. R.; Weitz, D. A.; Griffiths, A. D. Lab Chip 2009, 9, 18501858.

(33) Baker, N. R. Annu. Rev. Plant Biol. 2008, 59, 89-113.

(34) Xia, Y.; Whitesides, G. M. Angew. Chem., Int. Ed. 1998, 37, 550575.

(35) Siegel, A. C.; Bruzewicz, D. A.; Weibel, D. B.; Whitesides, G. M. Adv. Mater. 2007, 19, 727-733.

(36) Gorman, D. S.; Levine, R. P. Proc. Natl. Acad. Sci. U. S. A. 1965, 54, 1665-1669.

(37) Merchant, S. S.; Allen, M. D.; Kropat, J.; Moseley, J. L.; Long, J. C.; Tottey, S.; Terauchi, A. M. Biochim. Biophys. Acta, Mol. Cell Res. 2006, 1763, 578-594.

(38) Castenholz, R. W. Methods Enzymol. 1988, 167, 68-93.

(39) Haight, F. A. Handbook of the Poisson Distribution; John Wiley \& Sons: New York, 1967.

(40) Hill, E. K.; Mello, A. J. d. Analyst 2000, 125, 1033-1036.

(41) Msanne, J.; Xu, D.; Konda, A. R.; Casas-Mollano, J. A.; Awada, T.; Cahoon, E. B.; Cerutti, H. Phytochemistry 2012, 75, 50-59. 